Short communication

\title{
Ultra-highly sensitive and wash-free bio-detection of H5N1 virus by immunomagnetic reduction assays
}

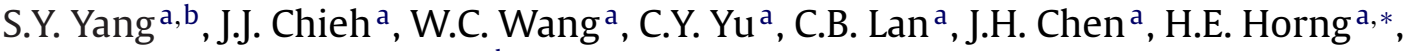 \\ Chin-Yih Hong ${ }^{c, *}$, H.C. Yang ${ }^{\mathrm{d}, *}$, Wilber Huang ${ }^{\mathrm{e}}$ \\ a Institute of Electro-optical Science and Technology, National Taiwan Normal University, Taipei 116, Taiwan \\ b MagQu Co., Ltd., Sijhih City, Taipei County 221, Taiwan \\ c Department of Mechanical Engineering, Nan-Kai Institute of Technology, Nan-tau 542, Taiwan \\ d Department of Physics, National Taiwan University, Taipei 106, Taiwan \\ e Abnova Inc., Taipei, Taiwan
}

Article history:

Received 27 March 2008

Received in revised form 8 July 2008

Accepted 22 July 2008

\section{Keywords:}

H5N1

Magnetic nanoparticles

SQUID

Immunomagnetic reduction

\begin{abstract}
A B S T R A C T
A platform for assaying avian influenza H5N1 viruses that involves measuring the ac immunomagnetic reduction of a magnetic reagent mixed with a detected sample is developed. The magnetic reagent contained magnetic nanoparticles coated with antibodies. To achieve an ultra-high sensitivity assay, a system utilizing a high-transition-temperature superconducting quantum interference device was used to sense the immunomagnetic reduction of the reagents. The results confirmed the ultra-high sensitivity of the immunomagnetic reduction assay on H5N1.
\end{abstract}

C 2008 Published by Elsevier B.V.
By binding antibodies onto magnetic nanoparticles dispersed in water, magnetic nanoparticles are able to mark specific bio-molecules. This feature makes bio-functionalized magnetic nanoparticles useful in bio-medicine. For example, bio-functionalized magnetic nanoparticles are used as contrast enhancers for magnetic resonance imaging, magnetic markers for cell sorting, and other purposes. In the late 1990s, another significant application for bio-functionalized magnetic nanoparticles was developed, called magnetically labeled diagnosis (MLD). In MLD, the concentration of the bio-molecules to be detected is determined by measuring variations in the magnetic properties of bio-functionalized magnetic nanoparticles. Several techniques have been proposed for MLD, such as magnetic relaxation (Kötitz et al., 1999; Yang et al., 2006), magnetic remanence (Enpuku et al., 1999), mixed-frequency magnetic susceptibility, saturated magnetization (Horng et al., 2006), nuclear magnetic resonance, and others. According to reports on these techniques, the highest sensitivity that has been achieved by a MLD has been sub-pico-gram $(\sim 0.1 \mathrm{pg})$ in the analyte (Enpuku et al., 1999), which was achieved with the magnetic remanence technique. However, there is still

\footnotetext{
* Corresponding authors. Tel.: +8862 29338260; fax: +886 286631954 .

E-mail addresses: phyfv001@scc.ntnu.edu.tw (H.E. Horng), cyhong@nkc.edu.tw (C.-Y. Hong), hcyang@phys.ntu.edu.tw (H.C. Yang).
}

interest in developing another alternative MLD technique to that can achieve higher sensitivities. For such an alternative technique, it would be better if only one type of antibody is necessary, and if the technique involves no bound/free particles separation processes (i.e., the technique is "wash-free"). In such a case, this alternative MLD technique would be very sensitive and very convenient, making the detection of biomolecules with ultra-low concentration feasible. Recently, an alternative technology for MLD was developed: ImmunoMagnetic Reduction (IMR) assay (Hong et al., 2007; Chieh et al., 2008), which has the advantages of requiring only a single antibody and wash-free. As a result, it seems to be a promising candidate for the convenient diagnosis of biomolecules. The mechanism of the IMR developed is described briefly as follows.

In IMR, the reagent is a solution containing homogeneously dispersed magnetic nanoparticles, which have been coated with hydrophilic surfactants and antibodies. Under external multiple ac magnetic fields, the magnetic nanoparticles oscillate with the multiple ac magnetic fields via magnetic interaction. Thus, the reagent under external multiple ac magnetic fields demonstrates a magnetic property, called multiple-frequency ac magnetic susceptibility $\chi_{\mathrm{ac}}$. Using the antibodies on the outermost shell, magnetic nanoparticles associate with the magnetically labeled bio-molecules to be detected. Due to this association, the magnetic nanoparticles become either larger or clustered, as shown schemat- 


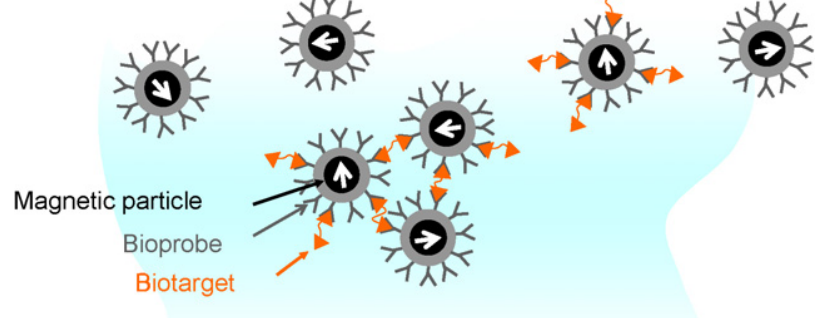

Fig. 1. Scheme for the association between the to-be-detected bio-molecules and magnetic nanoparticles coated with antibody. The magnetic nanoparticles become larger or clustered due to binding with the to-be-detected bio-molecules.

ically in Fig. 1. The response of these larger/clustered magnetic nanoparticles to the external multiple ac magnetic fields is much less than that of the original, individual magnetic nanoparticles. Thus, the $\chi_{\mathrm{ac}}$ of the reagent reduces due to the association between the magnetic nanoparticles and the detected bio-molecules. This is why the method is referred to as immunomagnetic reduction (IMR) assay.

Since IMR detects the change in the ac magnetic signals of samples, its sensitivity can be enhanced by using an ultra-sensitive sensor to probe the changes in the ac magnetic signals. Superconducting quantum interference devices (SQUIDs) are promising candidates as sensors in IMR systems, because SQUIDs are the most sensitive sensors available for probing magnetic signals, and their the changes. Usually, the noise level of a high- $T_{\mathrm{c}}$ SQUID is $10 \mathrm{fT} / \mathrm{Hz}^{1 / 2}$, whereas the signal generated by a magnetic nanoparticle is around $10 \mathrm{pT} / \mathrm{Hz}^{1 / 2}$. Thus, a SQUID system is suited for use as a sensor for an IMR with ultra-high sensitivity.

Fig. 2 depicts the scheme for the SQUID-based IMR system used here (XacPro-S101, MagQu Co., Ltd.). Briefly, the system consisted of three parts: the sample part, the SQUID part, and the electronic part. The sample part was composed of two excitation coils driven by two independent function generators to provide the sample with two ac excitation magnetic fields having the frequencies $f_{1}$ and $f_{2}$, respectively. The field strength for each excitation field is few gauss's. The sample was located inside one section of the pick-up coil, which was constructed of two sections of coils wired in opposite directions. The ac magnetization of a sample under the actions of the two excitation magnetic fields was detected by the pick-up coil. This signal was then guided to the SQUID part of the system via a fluxtransfer line made of $C u$ wire. The SQUID part consisted of a high- $T_{C}$ rf SQUID magnetometer, which was immersed in liquid nitro-

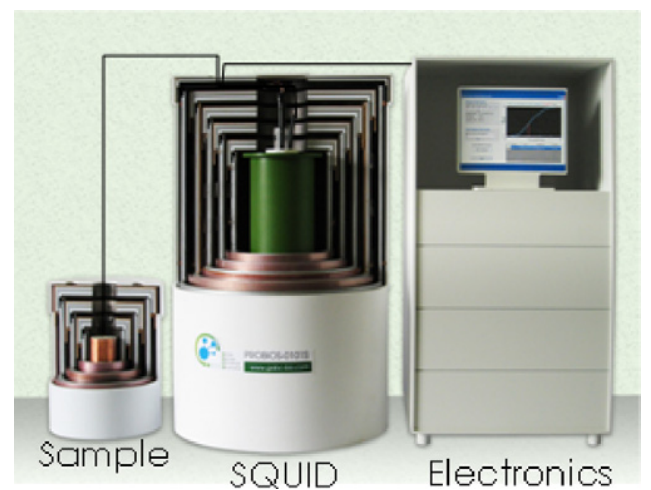

Fig. 2. Scheme of the SQUID-based IMR system assisted with flux-transfer technology. gen inside a dewar. The dewar was situated inside a magnetically shielded box and an rf shielded room (MAS-C5, MagQu) to prevent the SQUID from being affected by environmental low- or highfrequency noises. With this setup, the SQUID was not disturbed by the two excitation fields, because the excitation fields were far away from the SQUID. Thus, the system is very stable, making it suitable for long-time operation. SQUID electronics and read-out electronics were used for the SQUID to detect the ac magnetic flux generated originally by the sample at the sample part of the IMR system. The output voltages from the electronics were fed into a spectrum analyzer.

Assays on inactive H5N1 virus, pure H5N1 protein, pure H1N1 protein, and pure $\mathrm{H} 3 \mathrm{~N} 1$ protein were conducted individually. Before mixing the magnetic reagent with a detected samples, the 100$\mu \mathrm{l}, 0.3$-emu/g magnetic reagent injected into a glass tube, which is a container for reagent, at the target frequency $m f_{1}+n f_{2}$ was measured using the SQUID system, where $m$ and $n$ are non-zero integers. According to the specifications of XacPro-S101, $f_{1}$ and $f_{2}$ exist within the range from hundreds of $\mathrm{Hz}$ to tens of $\mathrm{kHz}$, depending on the particle size and coil characterizations. $f_{1}$ and $f_{2}$ were adjusted to achieve a maximum signal for $\chi_{\mathrm{ac}}$ of a sample at the target frequency $m f_{1}+n f_{2}$. Usually, $m$ and $n$ are 1,2 , or 3 .

After optimizing XacPro-S101, the IMR signal of a detected sample is measured. To do this, $20-\mu \mathrm{l}$ sample containing detected molecules was added into a $100-\mu$ l magnetic reagent which was already in a glass tube, followed by mixing well the solution using a Vortex for $2 \mathrm{~min}$. At this moment, immuno complex of detecedmolecule-anti-H5N1-magnetic nanoparticles was not formed. The $\chi_{\mathrm{ac}}$ of the mixture at target frequency was measured and denoted as $\chi_{\text {ac,o }}$. Then, the mixture of $100-\mu l$ magnetic reagent and $20-$ $\mu$ l sample containing detected molecules was kept at $25^{\circ} \mathrm{C}$ for incubating the immuno complex of detected-molecule-anti-H5N1magnetic nanoparticles. After incubation, the $\chi_{\mathrm{ac}}$ was measured again. This is denoted as $\chi_{\mathrm{ac}, \phi}$, where $\phi$ refers to the concentration of the detected molecules. Experimentally, the $\chi_{\mathrm{ac}, \phi}$ was found to be less than $\chi_{\mathrm{ac}, o}$. Then, a parameter $\Delta \chi_{\mathrm{ac}} / \chi_{\mathrm{ac}, \mathrm{o}}$ is defined as an indicator for the concentration/amount of detected molecules, where $\Delta \chi_{\mathrm{ac}} \mid \chi_{\mathrm{ac}, \mathrm{o}} \equiv\left(\chi_{\mathrm{ac}, 0}-\chi_{\mathrm{ac}, \phi}\right) / \chi_{\mathrm{ac}, \mathrm{o}} \times 100 \%$. In the experiment, the mean diameter of magnetic nanoparticles before and after incubation were detected by using dynamic light scattering technology, it was found that the mean diameter of magnetic nanoparticles changes from 50 to $200 \mathrm{~nm}$, or larger, depending on the concentration of to-be-detected molecules.

The values of $\Delta \chi_{\mathrm{ac}} / \chi_{\mathrm{ac}, \mathrm{o}}$ as a function of inactive virus were plotted with (-) in Fig. 3 in the coordinates of the left $y$ axis and the lower $x$ axis. It is noteworthy that the original concentration (referred as 1 at the lower $x$ axis) of the inactive virus is unknown. The numbers in the lower $x$ axis refer to the dilution ratios, i.e. $10^{3}$ denotes the concentration of one over thousand as the original concentration. To identify the sensitivity in terms of H5N1-protein concentration, the $\Delta \chi_{\mathrm{ac}} / \chi_{\mathrm{ac}, \mathrm{o}}$ as a function of the concentration of pure H5N1 protein was detected, and is plotted with $(x)$ in Fig. 3 in the coordinates of the left $y$ axis and the upper $x$ axis. It is clear that the sensitivity for assaying H5N1 via SQUID-based IMR is about $5 \mathrm{ppt}(=5 \mathrm{pg} / \mathrm{ml})$. Furthermore, the H5N1-protein reagent, i.e. magnetic nanoparticles coated with anti-H5N1 (Abnova), is used to assay H1N1 and H3N1 at the concentration of 200 ppt. The results are plotted with $(\boldsymbol{\Delta})$ and $(\boldsymbol{\square})$, respectively, for H1N1 and H3N1 in Fig. 3 in the coordinates of the left $y$ axis and the upper $x$ axis. Obviously, $\Delta \chi_{\text {ac }} / \chi_{\text {ac,o }}$ 's for H1N1 and H3N1 proteins via SQUID-based IMR with H5N1-protein reagent coincide with the noise level of the IMR system. This means that the anti-H5N1 coated magnetic nanoparticles can not significantly associate with $\mathrm{H} 1 \mathrm{~N} 1$ and H3N1 proteins. Thus, the SQUID-based IMR shows high-level specificity in assay. 


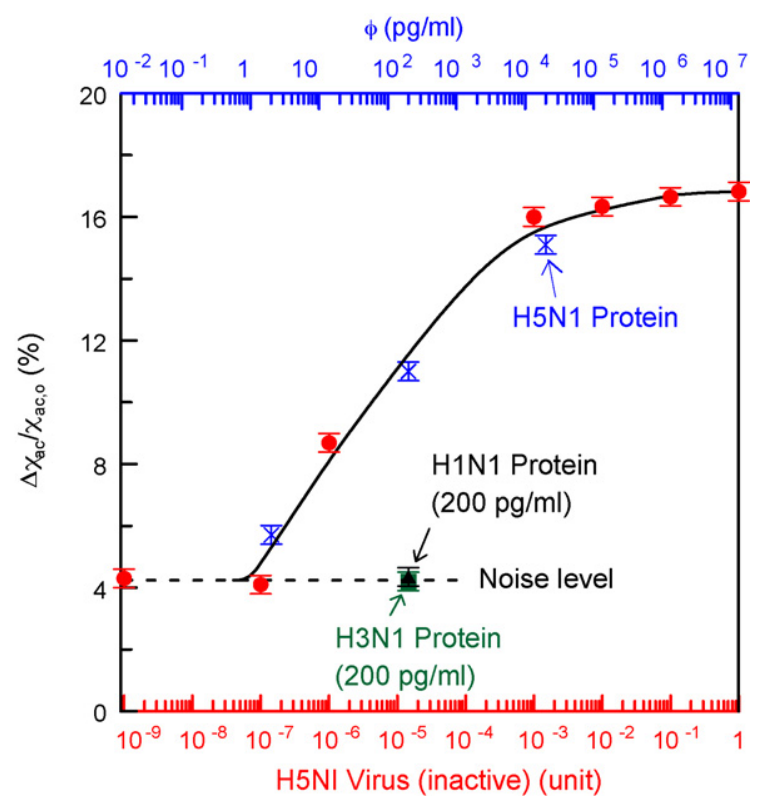

Fig. 3. $\Delta \chi_{\mathrm{ac}} / \chi_{\mathrm{ac}, \mathrm{o}}$ versus inactive virus concentration with H5N1 protein $(\bullet)$ in the coordinates of the left $y$ axis and the lower $x$ axis. $\Delta \chi_{\text {ac }} / \chi_{\mathrm{ac}, \mathrm{o}}$ versus pure H5N1 protein is plotted with $(x)$ in the coordinates of the left $y$ axis and the upper $x$ axis. $\Delta \chi_{\text {ac }} / \chi_{\text {ac,oo }}$ 's for 200 -ppt H1N1 and H3N1 proteins, respectively, are plotted with ( $\left.\mathbf{\Delta}\right)$ and $(\mathbf{\square})$ in the coordinates of the left $y$ axis and the upper $x$ axis.

In conclusion, a high- $T_{\mathrm{c}}$ SQUID-based system is utilized for H5N1 virus detection by an immunomagnetic reduction assay. In addition to the ultra-high sensitivity, high specificity was demonstrated by using SQUID-based IMR for wash-free assays. Therefore, SQUIDbased IMRs are a promising candidate for use in diagnosing and detecting viruses at ultra-low levels of concentration.

\section{Acknowledgement}

This work was supported by the National Science Council of Taiwan under Grant Nos. 95-2120-M-003-001, 95-2112-M-003017-MY2, and 95-2752-M-002-016-PAE.

\section{References}

Chieh, J.J., Yang, S.Y., Jian, Z.F., Wang, W.C., Horng, H.E., Yang, H.C., Hong, C.-Y., 2008 Hyper-high-sensitivity wash-free magnetoreduction assay on biomolecules using high- $T_{\mathrm{c}}$ superconducting quantum interference devices. J. Appl. Phys. 103, 14703-1-14703-6.

Enpuku, K., Minotani, T., Gima, T., Kuroki, Y., Itoh, Y., Yamashita, M., Katakura, Y., Kuhara, S., 1999. Detection of magnetic nanoparticles with superconducting quantum interference device (SQUID) magnetometer and application to immunoassays. Jpn. J. Appl. Phys. 38, L1102-L1105.

Hong, C.-Y., Chen, W.H., Chien, C.F., Yang, S.Y., Horng, H.E., Yang, L.C., Yang, H.C., 2007. Wash-free immunomagnetic detection for serum through magnetic susceptibility reduction. Appl. Phys. Lett. 90, 74105-1-74105-3.

Horng, H.E., Yang, S.Y., Hong, C.-Y., Liu, C.M., Tsai, P.S., Yang, H.C., Wu, C.C., 2006 Biofunctionalized magnetic nanoparticles for high-sensitivity immunomagnetic detection of human C-reactive protein. Appl. Phys. Lett. 88, 252506-1-2525063.

Kötitz, R, Weitschies, W. Trahms, L, Brewer W Semmler W 1999. Determination of the binding reaction between avidin and biotin by relaxation measurements of magnetic nanoparticles. J. Magn. Magn. Mater. 194, 62-68.

Yang, H.C., Yang, S.Y., Liao, S.H., Fang, G.L., Huang, W.H., Liu, C.H., Horng, H.E., Hong C.-Y., 2006. Magnetic relaxation measurement in immunoassay using hightransition-temperature superconducting quantum interference device system. J. Appl. Phys. 99, 124701-124705. 